\title{
NUMERICAL RESEARCH OF NONLINEAR VIBRATIONS OF ISOTROPIC VISCOELASTIC PLATES WITH VARIABLE RIGIDITY BY THE METHOD OF COMPUTER SIMULATION
}

\author{
Rustam Abdikarimov \\ Tashkent, Uzbekistan
}

\section{ABSTRACT}

In the given work the problem about vibrations of a isotropic viscoelastic plate with variable rigidity in geometrically nonlinear statement is considered. With the help of BubnovGalerkin method the problem is reduced to the decision of system of nonlinear ordinary integro-differential equations of Volterra's type. The numerical method based on the use of quadrature formulas is applied for the decision of the received system at weakly singular kernel of KoltunovRzhanitsin's. Influences of viscoelastic properties of a material, geometrical characteristics, and also dependences of change of thickness of a plate on process of vibrations are investigated.

\section{UDC CODE \& KEYWORDS}

- UDC: 539 - Viscoelastic Plate - Variable Thickness Nonlinear Vibrations $\square$ Bubnov-Galerkin method $\square$ Relaxation Kernel.

\section{INTRODUCTION}

It is well known that the majority of composite materials possess pronounced viscoelastic properties [1]. Wide industrial application of new materials with viscoelastic properties and analysis of their dynamic behavior indicate an important effect of heterogeneities on their strength. For the description of processes of deformation of viscoelastic materials various models of the hereditary theory of viscoelasticity are used. Necessity of the account of viscoelastic properties of materials for engineering calculations was reflected in occurrence of a plenty more or less simple theories. There is a number of the works devoted to research of behavior of viscoelastic systems. In these works of a problem have been considered or with the help of differential model Voight, or Boltzmann-Volterra's integral model, where exponential kernels were taken as kernels of relaxation, although they could not describe the real processes occurring in shells and plates at zero time. These models can be used only for the description of the separate phenomena in viscoelastic shells.

The purpose of the given work is research of nonlinear vibrations of viscoelastic plate with variable rigidity.

Mathematic model of the problem about nonlinear vibrations of viscoelastic plate with variable rigidity.

Consider a rectangular viscoelastic plate of variable thickness $h=h(x, y)$ with sides $a$ and $b$, made of homogeneous isotropic material (fig.1).

Figure 1: Rectangular viscoelastic plate

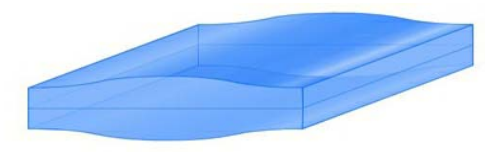

Source: Author
For construction of mathematical model of the problem about nonlinear vibrations of viscoelastic isotropic plate in geometrically nonlinear statement on Kirchhoff-Love's kinematic hypothesis, physical dependence between

stresses $\sigma_{x}, \sigma_{y}, \tau_{x y}$ and strains $\varepsilon_{x}, \varepsilon_{y}, \gamma_{x y}$ is taken in the form $[1,2]$ :

$$
\sigma_{x}=\frac{E}{1-\mu^{2}}\left(1-I^{*}\right)\left(\varepsilon_{x}+\mu \varepsilon_{y}\right), x \leftrightarrow y, \quad \tau_{x y}=\frac{E}{2(1+\mu)}\left(1-\Gamma^{*}\right) \gamma_{x y}, \quad(1)
$$

Where $\Gamma^{*}-$ the integral operator with the kernel of relaxation $\left.\Gamma(t): \Gamma^{*} \varphi=\int_{0}^{1} \Gamma(t-\tau) \varphi(\tau)\right) d \tau ; \quad \mu$ is Poisson's ratio; $E$ is the elasticity modulus. Hereinafter the symbol $(x \leftrightarrow y)$ shows that all the remaining equations not explicitely written are obtained by circular substitution of indexes.

The relationship between strains $\varepsilon_{x}, \varepsilon_{y}, \gamma_{x y}$ and displacements $u, v, w$ in directions $x, y, z$ is obtained in [3]:

$$
\varepsilon_{x}=\frac{\partial u}{\partial x}+\frac{1}{2}\left(\frac{\partial w}{\partial x}\right)^{2}, \quad \varepsilon_{y}=\frac{\partial v}{\partial y}+\frac{1}{2}\left(\frac{\partial w}{\partial y}\right)^{2}, \gamma_{x y}=\frac{\partial u}{\partial y}+\frac{\partial v}{\partial x}+\frac{\partial w}{\partial x} \frac{\partial w}{\partial y} \text { (2) }
$$

We express the bending and twisting moments of the element of the plate as follows:

$$
M_{x}=-D\left(1-I^{*}\right)\left(\frac{\partial^{2} w}{\partial x^{2}}+\mu \frac{\partial^{2} w}{\partial y^{2}}\right), x \leftrightarrow y, H=-D(1-\mu)\left(1-I^{*}\right) \frac{\partial^{2} w}{\partial x \partial y^{\prime}}, \quad \text { (3) }
$$

where $D=\frac{E h^{3}(x, y)}{12\left(1-\mu^{2}\right)}$ - variable cylindrical rigidity.

When deriving the equations of motion of the element of the viscoelastic isotropic plate, we shall proceed from equations [3]:

$$
\begin{gathered}
\frac{\partial N_{x}}{\partial x}+\frac{\partial N_{x y}}{\partial y}+p_{x}-\rho h \frac{\partial^{2} u}{\partial t^{2}}=0, \frac{\partial N_{x y}}{\partial x}+\frac{\partial N_{y}}{\partial y}+p_{y}-\rho h \frac{\partial^{2} v}{\partial t^{2}}=0 \\
\frac{\partial M_{x}}{\partial x^{2}}+\frac{\partial^{2} M_{y}}{\partial y^{2}}+2 \frac{\partial^{2} H}{\partial x \partial y}+\frac{\partial}{\partial x}\left(N_{x} \frac{\partial w}{\partial x}+N_{x y} \frac{\partial w}{\partial y}\right)+ \\
+\frac{\partial}{\partial y}\left(N_{x y} \frac{\partial w}{\partial x}+N_{y} \frac{\partial w}{\partial y}\right)+q-\rho h \frac{\partial^{2} w}{\partial t^{2}}=0
\end{gathered}
$$

Here $N_{x}, N_{y}$ and $N_{x y}$ are the forces referred to unit of length of section of the plate:

$$
N_{x}=\sigma_{x} h, \quad x \leftrightarrow y, \quad N_{x y}=\gamma_{x y} h
$$


$p_{x}, p_{y}$ and $q$ are intensity of stated external load enclosed to the element in directions $x, y$ and $z$ accordingly. With the account (1) substituting (3) and (5) in (4) we shal receive the following system of the nonlinear integrodifferential equations with variable coefficients:

$$
\begin{aligned}
& \left(1-I^{*}\left[h\left(\frac{\partial \varepsilon_{x}}{\partial x}+\mu \frac{\partial \varepsilon_{y}}{\partial x}+\frac{1-\mu}{2} \frac{\partial \gamma_{y}}{\partial y}\right)+\frac{\partial h}{\partial x}\left(\varepsilon_{x}+\mu \varepsilon_{y}\right)+\frac{1-\mu}{2} \frac{\partial h}{\partial y} \gamma_{w}\right]+\right. \\
& +\frac{1-\mu^{2}}{E} p_{x}-\rho h \frac{1-\mu^{2}}{E} \frac{\partial^{2} u}{\partial^{2}}=0 \\
& \left(1-\Gamma^{*}\left[h\left(\frac{\partial \varepsilon_{y}}{\partial y}+\mu \frac{\partial \varepsilon_{x}}{\partial y}+\frac{1-\mu}{2} \frac{\partial \gamma_{w}}{\partial x}\right)+\frac{\partial h}{\partial y}\left(\varepsilon_{y}+\mu \varepsilon_{x}\right)+\frac{1-\mu}{2} \frac{\partial h}{\partial x} \gamma_{w}\right]+\right. \\
& +\frac{1-\mu^{2}}{E} p_{y}-\rho h \frac{1-\mu^{2}}{E} \frac{\partial^{2} v}{\partial t^{2}}=0 \\
& \left(1-I^{*}\right)\left\{\left[D \nabla^{4} w+2 \frac{\partial D}{\partial x} \frac{\partial}{\partial x} \nabla^{2} w+2 \frac{\partial D}{\partial y} \frac{\partial}{\partial y} \nabla^{2} w+\nabla^{2} D \nabla^{2} w-\right.\right. \\
& \left.-\left(1-\mu\left(\frac{\partial^{2} D}{\partial x^{2}} \frac{\partial^{2} w}{\partial y^{2}}-2 \frac{\partial^{2} D}{\partial x \partial y} \frac{\partial^{2} w}{\partial x \partial y}+\frac{\partial^{2} D}{\partial y^{2}} \frac{\partial^{2} w}{\partial x^{2}}\right)\right]\right\}+ \\
& +\frac{E h}{1-\mu^{2}}\left\{\frac{\partial}{\partial x}\left[\frac{\partial w}{\partial x}\left(1-\Gamma^{*}\right)\left(\varepsilon_{x}+\mu \varepsilon_{y}\right)+\frac{1-\mu}{2} \frac{\partial w}{\partial y}\left(1-\Gamma^{*}\right) \gamma_{x y}\right]+\right. \\
& \left.+\frac{\partial}{\partial y}\left[\frac{\partial w}{\partial y}\left(1-\Gamma^{*}\right)\left(\varepsilon_{y}+\mu \varepsilon_{x}\right)+\frac{1-\mu}{2} \frac{\partial w}{\partial x}\left(1-\Gamma^{*}\right) / \gamma_{y}\right]\right\}+ \\
& +\frac{E}{1-\mu^{2}} \frac{\partial h}{\partial x}\left[\frac{\partial w}{\partial x}\left(1-I^{*}\right)\left(\varepsilon_{x}+\mu \varepsilon_{y}\right)+\frac{1-\mu}{2} \frac{\partial w}{\partial y}\left(1-\Gamma^{*}\right) \gamma_{w}\right]+ \\
& +\frac{E}{1-\mu^{2}} \frac{\partial h}{\partial y}\left[\frac{\partial w}{\partial y}\left(1-I^{*}\right)\left(\varepsilon_{y}+\mu \varepsilon_{x}\right)+\frac{1-\mu}{2} \frac{\partial w}{\partial x}\left(1-\Gamma^{*}\right) \eta_{y}\right]+q-\rho h \frac{\partial^{2} w}{\partial^{2}}=0
\end{aligned}
$$

Let's notice, that the system of the nonlinear integrodifferential equations of motion of viscoelastic plate is the general from which in that specific case it is possible to receive the equations of motion of viscoelastic plates which thickness changes only in one direction, or on an axis $O x$, or on $O y$, and also viscoelastic plate of constant thickness

1. For viscoelastic isotropic plate at $h=h(x)$, we shall receive

$$
\begin{aligned}
& 1-I^{\cdot}\left[h\left(\frac{\partial \varepsilon_{x}}{\partial x}+\mu \frac{\partial \varepsilon_{y}}{\partial x}+\frac{1-\mu}{2} \frac{\partial \gamma_{y}}{\partial y}\right)+\frac{\partial}{\partial x}\left(\varepsilon_{x}+\mu \varepsilon,\right)\right]+\frac{1-\mu^{2}}{E} p_{x}-\rho h \frac{1-\mu^{2}}{E} \frac{\partial^{2} u}{\partial t^{2}}=0 \\
& \left(1-\Gamma^{*}\left[h\left(\frac{\partial \varepsilon_{y}}{\partial y}+\mu \frac{\partial \varepsilon_{x}}{\partial y}+\frac{1-\mu}{2} \frac{\partial \gamma_{y}}{\partial x}\right)+\frac{1-\mu}{2} \frac{\partial h}{\partial x} \gamma_{y y}\right]+\right. \\
& +\frac{1-\mu^{2}}{E} p_{y}-\rho h \frac{1-\mu^{2}}{E} \frac{\partial^{2} v}{\partial t^{2}}=0 \\
& \left(1-I^{*}\left[D \nabla^{4} w+2 \frac{\partial D}{\partial x} \frac{\partial}{\partial x} \nabla^{2} w+\frac{\partial^{2} D}{\partial x^{2}} \nabla^{2} w-(1-\mu) \frac{\partial^{2} D}{\partial x^{2}} \frac{\partial^{2} w}{\partial y^{2}}\right]+\right. \\
& +\frac{E h}{1-\mu^{2}}\left\{\frac{\partial}{\partial x}\left[\frac{\partial w}{\partial x}\left(1-\Gamma^{*}\right)\left(\varepsilon_{x}+\mu \varepsilon_{y}\right)+\frac{1-\mu}{2} \frac{\partial w}{\partial y}\left(1-\Gamma^{*}\right) \eta_{x y}\right]+\right. \\
& \left.+\frac{\partial}{\partial y}\left[\frac{\partial w}{\partial y}\left(1-\Gamma^{*}\right)\left(\varepsilon_{y}+\mu \varepsilon_{x}\right)+\frac{1-\mu}{2} \frac{\partial w}{\partial x}\left(1-\Gamma^{*}\right) \eta_{w}\right]\right\}+ \\
& +\frac{E}{1-\mu^{2}} \frac{\partial h}{\partial x}\left[\frac{\partial w}{\partial x}\left(1-I^{*}\right)\left(\varepsilon_{x}+\mu \varepsilon_{y}\right)+\frac{1-\mu}{2} \frac{\partial w}{\partial y}\left(1-I^{*}\right) y_{x}\right]+q-\rho h \frac{\partial^{2} w}{\partial^{2}}=0
\end{aligned}
$$

2. Similarly, at $h=h(y)$ we shall receive the following equations

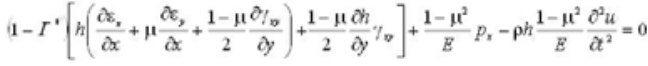

$$
\begin{aligned}
& \left(1-I^{*}\left[h\left(\frac{\partial \varepsilon_{y}}{\partial y}+\mu \frac{\partial \varepsilon_{x}}{\partial y}+\frac{1-\mu}{2} \frac{\partial \gamma_{y}}{\partial x}\right)+\frac{\partial h}{\partial y}\left(\varepsilon_{y}+\mu \varepsilon_{x}\right)+\frac{1-\mu}{2} \frac{\partial h}{\partial x} \gamma_{w}\right]+\right. \\
& +\frac{1-\mu^{2}}{E} p_{y}-\rho h \frac{1-\mu^{2}}{E} \frac{\partial^{2} v}{\partial t^{2}}=0 \\
& \left(1-I^{*}\left[D \nabla^{4} w+2 \frac{\partial D}{\partial y} \frac{\partial}{\partial y} \nabla^{2} w+\frac{\partial^{2} D}{\partial y^{2}} \nabla^{2} w-(1-\mu) \frac{\partial^{2} D}{\partial y^{2}} \frac{\partial^{2} w}{\partial x^{2}}\right]+\right. \\
& +\frac{E h}{1-\mu^{2}}\left\{\frac{\partial}{\partial x}\left[\frac{\partial w}{\partial x}\left(1-\Gamma^{*}\right)\left(\varepsilon_{x}+\mu \varepsilon_{y}\right)+\frac{1-\mu}{2} \frac{\partial w}{\partial y}\left(1-\Gamma^{*}\right) \gamma_{x y}\right]+\right. \\
& \left.+\frac{\partial}{\partial y}\left[\frac{\partial w}{\partial y}\left(1-\Gamma^{*}\right)\left(\varepsilon_{y}+\mu \varepsilon_{z}\right)+\frac{1-\mu}{2} \frac{\partial w}{\partial x}\left(1-\Gamma^{*}\right) \gamma_{x y}\right]\right\}+ \\
& \left.+\frac{E}{1-\mu^{2}} \frac{\partial h}{\partial y}\left[\frac{\partial w}{\partial y}\left(1-I^{*}\right) \varepsilon_{y}+\mu \varepsilon_{x}\right)+\frac{1-\mu}{2} \frac{\partial w}{\partial x}\left(1-I^{*}\right) \gamma_{y}\right]+q-\rho h \frac{\partial^{2} w}{\partial^{2}}=0
\end{aligned}
$$

3. At constant thickness ( $h=$ const ) we shall receive

$$
\begin{aligned}
& \left(1-I^{*}\left[h\left(\frac{\partial \varepsilon_{x}}{\partial x}+\mu \frac{\partial \varepsilon_{y}}{\partial x}+\frac{1-\mu}{2} \frac{\partial \gamma_{y}}{\partial y}\right)\right]+\frac{1-\mu^{2}}{E} p_{x}-\rho h \frac{1-\mu^{2}}{E} \frac{\partial^{2} u}{\partial^{2}}=0\right. \\
& \left(1-I^{*}\left[h\left(\frac{\partial \varepsilon_{y}}{\partial y}+\mu \frac{\partial \varepsilon_{x}}{\partial y}+\frac{1-\mu}{2} \frac{\partial \gamma_{w}}{\partial x}\right)\right]+\frac{1-\mu^{2}}{E} p_{y}-\rho h \frac{1-\mu^{2}}{E} \frac{\partial^{2} v}{\partial^{2}}=0\right. \\
& \left(1-I^{*}\right) D \nabla^{4} w+\frac{E h}{1-\mu^{2}}\left\{\frac{\partial}{\partial x}\left[\frac{\partial w}{\partial x}\left(1-I^{*}\right) \varepsilon_{x}+\mu \varepsilon_{y}\right)+\frac{1-\mu \partial w}{2} \frac{\partial w}{\partial y}\left(1-I^{*}\right) \gamma_{w}\right](9) \\
& \left.\left.+\frac{\partial}{\partial y}\left[\frac{\partial w}{\partial y}\left(1-I^{*}\right) \varepsilon_{y}+\mu \varepsilon_{x}\right)+\frac{1-\mu}{2} \frac{\partial w}{\partial x}\left(1-I^{*}\right) \gamma_{w}\right]\right\}+q-\rho h \frac{\partial^{2} w}{\partial^{2}}=0
\end{aligned}
$$

Thus, the mathematical model of the problem about vibrations of viscoelastic plates with variable rigidity in geometrically nonlinear statement is described by system of the integro-differential equations in partial derivatives of type (6) with boundary and initial conditions.

Calculation of nonlinear vibrations of a viscoelastic plate with variable rigidity. Bibnov-Galerkin method.

Let the viscoelastic plate with variable thickness be supported by hinges on all sides. The displacements of the plate we approximate functions:

$$
\begin{gathered}
u(x, y, t)=\sum_{n=1}^{M} \sum_{m=1}^{M} u_{n m}(t) \phi_{n m}(x, y), v(x, y, t)=\sum_{n=1}^{M} \sum_{m=1}^{M} v_{n m}(t) \varphi_{x m}(x, y) \\
w(x, y, t)=\sum_{n=1}^{N} \sum_{m=1}^{M} w_{n m}(t) \Psi_{n m}(x, y)_{(10)}
\end{gathered}
$$

where $\quad u_{n m}=u_{n m}(t), \quad v_{n m}=v_{n m}(t), \quad w_{n m}=w_{n m}(t)$ unknown functions of time; $\phi_{n m}(x, y), \quad \varphi_{n m}(x, y)$, $\psi_{n m}(x, y), \quad n=1,2, \ldots, N ; \quad m=1,2, \ldots, M \quad$ are the coordinate functions satisfying set boundary conditions of the problem.

Considering $p_{x}, p_{y}=0$, substituting (10) in the equation (6), thus applying Bubnov-Galerkin method and having entered the following dimensionless sizes

$$
\frac{u}{h_{0}}, \frac{v}{h_{0}}, \frac{w}{h_{0}}, \frac{x}{a}, \frac{y}{b}, \omega t, \frac{h}{h_{0}}, \lambda=\frac{a}{b}, \delta=\frac{b}{h_{0}}
$$


and keeping the previous notation for the dimensionless parameters $u_{n m}=u_{n m}(t), \quad v_{n m}=v_{n m}(t), \quad w_{n m}=w_{n m}(t)$ we obtain the following system of the basic resolving nonlinear integro-differential equations:

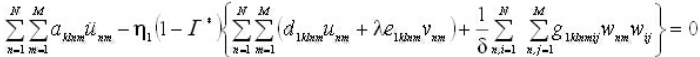

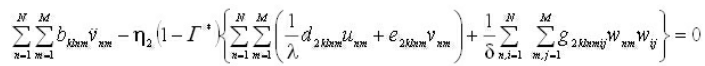

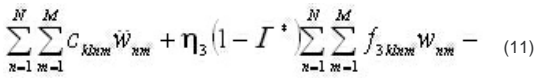

$$
\begin{aligned}
& -\eta_{3}\left\{\sum_{n, i=1}^{N} \sum_{m, j=1}^{M} w_{x m}\left(1-\Gamma^{*}\right)\left(d_{4 k \times m m_{j}} u_{i j}+e_{4 k \times m m i j} v_{i j}\right)+\right. \\
& \left.+\sum_{x, i, r=1}^{N} \sum_{m, j, j, 1}^{M} g_{k x m i v ;} w_{x m}\left(1-\Gamma^{*}\right) w_{i j} w_{r 3}\right\}=12 \eta_{3}\left(1-\mu^{2}\right) \lambda^{4} q_{k}
\end{aligned}
$$

where $h_{0}=h(0)=$ const $; \quad \eta_{1}=\frac{3 \delta^{2}}{\pi^{4} \lambda^{2}} ; \quad \eta_{2}=\frac{3 \delta^{2}}{\pi^{4}} ; \quad \eta_{3}=\frac{1}{4 \pi^{4} \lambda^{4}} ;$ other constant coefficients which are included in this system are connected to coordinate functions and their derivatives Integration of system (11) was carried out with the help of the numerical method based on the use of the quadrature formulas $[4,5]$. At calculation of problems we shall use the elementary and, at the same time, quite general weakly singular kernel of Koltunov-Rzhanitsin's with three rheological parameters $A, \beta$ and $\alpha$ in the form [2]:

$$
\Gamma(t)=A e^{-\beta t} t^{\alpha-1},(0<\alpha<1)
$$

Calculations were carried out on algorithmic language Delphi. As an example some special cases of this problem when thickness of the plate changes only in one direction, along an axis $O x$, on following are considered by laws:

$$
\begin{aligned}
& h=h(x)=1-\alpha^{*} x, \quad 0<\alpha^{*}<1 \\
& h=h(x)=1+\alpha^{*} x, \quad 0<\alpha^{*}<1 \\
& h=h(x)=1+0.3 \sin (2 r-1) \pi x
\end{aligned}
$$

where $\alpha^{*}, r-$ parameters of change of thickness.

Results of calculations, at various physical and geometrical parameters of the viscoelastic plate are resulted on Fig.2-7. Here, if not written otherwise, initial data were taken in the following form: $A=0.05 ; \quad \alpha=0.25 ; \quad \beta=0.05$; $\mu=0.3 ; \delta=25 ; u_{0}=0 ; v_{0}=0 ; w_{0}=0.01 ; q=0$; $\lambda=1 ; h=1-\alpha^{*} x ; \alpha^{*}=0.3$.

In Fig. 2 dependence of the deflection on time of the median point elastic ( $A=0$ - the curve 1$)$ and viscoelastic plates $(A=0.05,0.1$ - curves 2,3$)$ is submitted. As can be seen from the figure, the account of the viscoelastic properties of the material of the plate leads to damping of the vibration process.

Although the solution of elastic and viscoelastic problems at zero times does not differ from each other, as time unfolds, the viscoelastic properties have a more pronounced influence.

Influence of another rheological parameter $\alpha$ on the vibration process is shown on Fig.3. From figure it is visible, www.journals.cz that the increase in value of this parameter results in increase of amplitude and frequency of vibrations.

Figure 2: Dependence of the deflection on time at

$$
A=0 \text { (1), } 0.05 \text { (2), } 0.1 \text { (3) }
$$

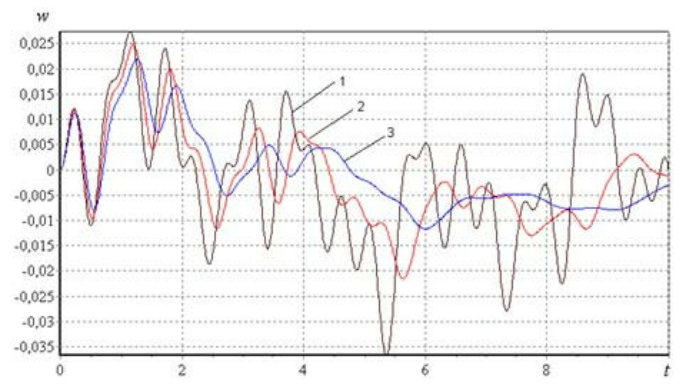

Source: Author

Figure 3: Dependence of the deflection on time at $\alpha=0.25 \square(1), 0.5(2), 0.75$ (3)

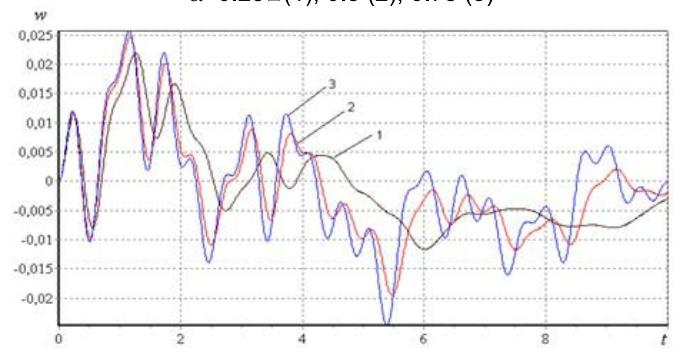

Source: Author

Further calculations show that the change of the third rheological parameter, viscosity $\beta(0<\beta<1)$, does not sufficiently influence the process of vibrations of the viscoelastic plate, once again confirming the statement on the inapplicability of the use of exponential kernels of relaxation in the design of dynamic problems of viscoelastic systems.

Change of thickness of the viscoelastic plate under the law (12), at equal volumes of plates with constant and variable thickness, results (Fig.4) in reduction of the maximal displacements.

Figure 4: Dependence of the deflection on time at $\alpha^{*}=0(1), 0.3(2), 0.5(3)$

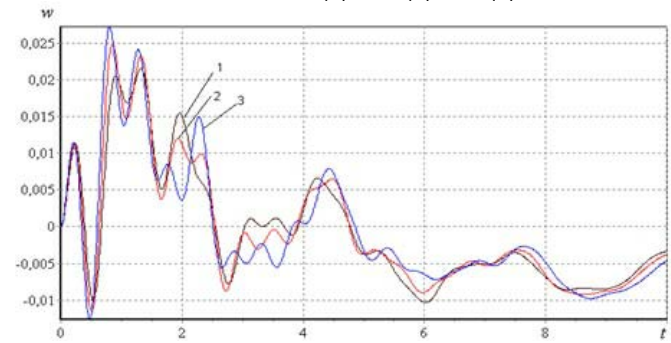

Source: Author

Many numerical experiments confirmed the same and for other kinds of dependences of change of thickness of the plate.

On Fig.5-7 spatial forms of vibrations of the viscoelastic plate are submitted at constant thickness (Fig.5), and also at the thickness changing on dependences (12) (Fig. 6) and (13) (Fig.7) during the initial moment of time. From the resulted figures it is visible, that the viscoelastic plate at constant 
thickness changes symmetrically (Fig. 5), and at variable thickness is asymmetric (Fig. 6 and 7).

Figure 5: The viscoelastic plate with constant thickness

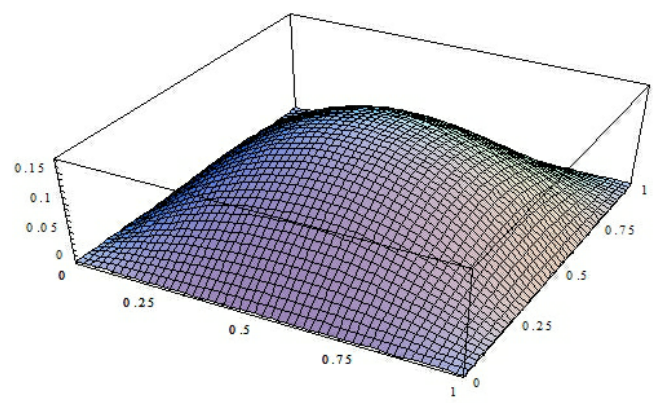

Source: Author

Figure 6: The viscoelastic plate with the thickness changing on dependence (12) at $\alpha^{*}=0.5$

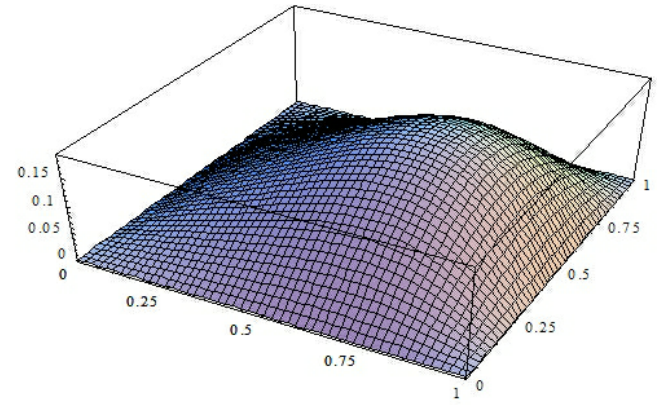

Source: Author

Figure 7: The viscoelastic plate with the thickness changing on dependence (13) at $\alpha^{*}=0.5$

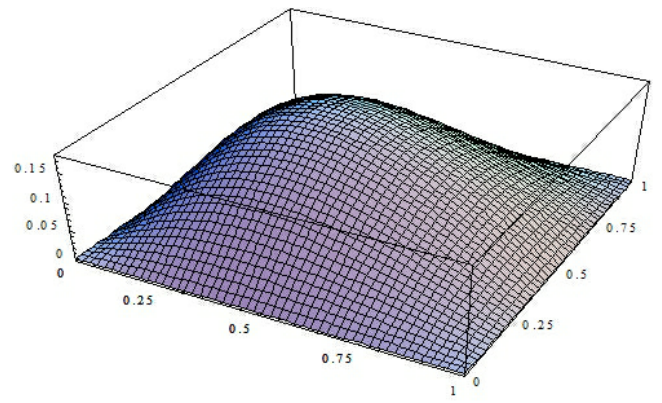

Source: Author

In each separately considered case convergence of BubnovGalerkin method is investigated. While determining the deflection values, the first seven harmonics $(N=7, M=1)$ were retained. Results show that a further increase in the number of components does not greatly influence the amplitude of vibrations of the viscoelastic plate.

Investigation of nonlinear problems of vibrations of viscoelastic plates with variable thickness shows that:

1. Predictably, the account of real properties of material of the plate, i.e. viscoelastic properties of material of the plate, results in reduction of amplitude of vibrations, and reduction of value of rheological parameter $\alpha$ results in reduction of frequency of vibrations. Rheological parameter $\beta$ does not influence process of vibrations.

2. On process of vibrations renders essential influence of dependence of change of thickness of the plate. Change of thickness of the viscoelastic plate at equal volumes of plates with constant and variable thickness results in reduction of the maximal displacements, and also redistribution of stresses.

\section{REFERENCES}

1. Koltunov M.A. Creep and relaxation, Visshaya shkola, Moscow, 1976.

2. Il'yushin A.A., Pobedrya B.E. Fundamentals of the mathematical theory of thermoviscoelasticity, Nauka, Moscow, 1970.

3. Volmir A.S. The nonlinear dynamics of plates and shells, Nauka, Moscow, 1972.

4. Badalov F.B., Eshmatov Kh., Yusupov M. About some methods of the decision of systems integro-differential equations meeting in problems viscoelasticity. Soviet Applied Mathematics and Mechanics [Translated from Prikladnaya Matematika i Mekhanika], 1987; 51: 867871.

5. Verlan A.F., Abdikarimov R.A., Eshmatov Kh. Numerical simulation of nonlinear problems of dynamics of viscoelastic systems with variable rigidity. Electronic Modeling, 2010; 2(32): 3-13. 